\title{
Language Variation and Linguistic Invariants
}

\author{
Edward L. Keenan, Edward P. Stabler \\ Department of Linguistics, University of California, Los Angeles, 90095-1543, USA
}

\begin{abstract}
Human languages are diverse and so precise statements of common properties must abstract away from specifics of particular languages. We note several abstract and absolute 'Type 1' universals and present some new ones built on a notion of 'structural invariant' that applies equally to constituent structure and individual grammatical morphemes. Crucially, these universals may be realized in structurally diverse ways.
\end{abstract}

Keywords: Language universal; invariant; anaphora; binding theory

\section{Introduction}

Evans and Levinson (2009, henceforth E\&L) argue that "languages differ so fundamentally from one another at every level of description (sound, grammar, lexicon, meaning) that it is very hard to find any single structural property which they share" (p.429), as evidenced by the many particular proposals that have been challenged by later discoveries (Box 1, p.431). But is the Book of Language Universals as thin as E\&L say, and is their attitude to counterexamples to universal claims a constructive one? We disagree on both counts. We focus here on the former, but note first some known objections to the latter which we feel bear repeating.

In general progress in a science is spurred on, not defeated, by counterexamples (a point supported by previous commentators). This will mean that at any given point in our scientific endeavor we have to hold incompatible data in our minds until we understand the data better, modify the theory (or both). What a loss to science and human understanding had we rejected the Mendelian claims of segregation and independence of heritable traits faced with the "obvious" counterexamples of "blends" such as mongrel dogs produced by mating ones of distinct species. Similarly Galileo saw with striking clarity that a projectile like a cannonball or a ball dropped from the mast of a ship instantiated two "motions" at once, their vector sum determining the trajectories of the balls (per the parallelogram law we learn in school). We don't abandon this idea just because today we know that other (lesser) factors are involved - not just "performance" factors like different cross-wind velocities in different experiments but principled ones like the curvature of the Earth and the variation of the speed of rotation with latitude (Asimov, 1966, 40). Pursuing the Mendelian and Galilean abstract structural analyses despite problematic cases at every step of the way has increased, and still increases, our understanding. Equally we have learned an enormous amount (Büring, 2005; König and Gast, 2008) about the anaphora systems

Email addresses: Edward.Keenan1@gmail.com (Edward L. Keenan), stabler@ucla.edu (Edward P. Stabler) 
of human languages over the last 25 years despite (as we illustrate below) the failure of Principle A with its attendant c-command requirement on antecedents of anaphors.

\section{Abstract universals}

E\&L equate "abstraction" with "unobservables", claiming that "Abstractness has a cost: the more unverifiable unobservables, the greater the explanatory payoff we expect". This notion of abstractness does not coincide with ours, nor, we feel, with that used in science in general. Abstracting away from the particular details of each situation is essential in all sciences, and is not the same as positing "unverified unobservables". E\&L neglect a long tradition of abstract claims about language which do not refer to unobservables or even to specific grammatical categories or elements of a particular grammar. Some examples: human languages are compositional (Frege, 1923); all languages are infinite; all express predicate-argument relations; all are recursive (Chomsky, 1965, p.62) and the much stronger claim of Joshi (1985) that they are all "mildly context sensitive". Joshi's claim has been challenged (Rambow, 1994; Michaelis and Kracht, 1997; Stabler, 1997; Kobele, 2006), but we know of no serious arguments against the slightly weaker view that all human languages are properly included in the of context sensitive languages defined by 'parallel multiple context free' (PMCF) grammars (Seki et al., 1991; Kaji et al., 1994) - a claim still much stronger than Chomsky's. The claim that human languages are definable by such grammars is substantial, since, for example, the languages they define can be efficiently recognized. And again this abstract claim does not involve positing unobservables. It is related to the "non-counting" universal noted in Newmeyer (2005, (2.a), p.4). ${ }^{1}$, who in fact proposes 25 universals, none of which are considered by E\&L. And in recent work, Keenan and Stabler $(2003,2005)$ show how to define, with mathematical rigor, invariant properties of language (subject to empirical validation or refutation) that generalize over structural diversity such as constituent structure vs. case marking (cited by E\&L as problematic for usual claims of structural universality).

\section{Structure and invariants}

Different grammatical traditions - LFG, HPSG, Relational Grammar, Minimalism - assign different structures to even simple sentences such as Dana is laughing. But they would agree that the sentence Dana is laughing and the sentence Robyn is crying have the same structure. Our intuition is that substituting Robyn for Dana in the first expression yielding Robyn is laughing preserves the structure of the original. Similarly replacing laugh with cry is structure preserving, whence Robyn is crying has the same structure as Dana is laughing. Representing substitutions as functions replacing expressions by expressions and writing $L(G)$ for the set of expressions generated by a grammar $\mathrm{G}$, we generalize in a mathematically standard way to say that function $h$ from a language $\mathrm{L}(\mathrm{G})$ onto $\mathrm{L}(\mathrm{G})$ is structure preserving if it is one to one and does not change how $\mathrm{G}$ builds expressions. So if an expression $z$ is derived by a rule R (say Merge, or Move, or some more structure specific one) from expressions $x$ and $y$, then $h(z)$, the expression that $h$ maps $z$ to, must be derived from $h(x)$ and $h(y)$ by $\mathrm{R}$ as well. So $z$ and $h(z)$ are derived in exactly the

\footnotetext{
${ }^{1}$ Non-counting languages have been rigorously defined (Crespi-Reghizzi et al., 1978) with notions closely related to the so-called 'pumping lemmas' for subclasses of the context sensitive languages (Seki et al., 1991; Kanazawa, 2009).
} 
same way. Such a structure preserving map is called an automorphism, and two expressions will be said to have the same structure iff there is an automorphism which maps one to the other.

Crucially now, we define an expression itself to be (structurally) invariant iff all the automorphisms map that expression to itself. That is, you cannot replace it by any other expression preserving how expressions are derived. In model grammars we have defined, the nominative and accusative case markers in (our) Korean are invariant. In our model of Toba Batak (W. Austronesian) voice affixes are invariant. So note that morphology can be structurally invariant. In general we expect that expressions linguists call "functional expressions" or "grammatical morphemes" will be formally invariant in all empirically adequate, explicit grammars.

The notion of invariant applies as well to properties, like is a VP, is an anaphor, and to relations, like c-commands or is a possible antecedent of. Thus a property $\mathrm{P}$ of expressions is invariant iff $\mathrm{P}$ holds of an expression $x$ iff it holds of $h(x)$, for all automorphisms $h$. (This just says that $h[\mathrm{P}]=\mathrm{P}$, for all automorphisms $h$.) We expect for example that in an explicit grammar for English the property of being a VP is invariant. You cannot map a VP to a non-VP preserving how expressions are built. Similarly a relation $\mathrm{R}$ is invariant iff whenever $x \mathrm{R} y$ then $h(x) \mathrm{R} h(y)$, for all automorphisms $h$, that is, $h[\mathrm{R}]=\mathrm{R}$. So in general the (structural) invariants of a grammar $\mathrm{G}$ are those linguistic objects - expressions, properties of expressions, relations between expressions, ... which are mapped to themselves by the syntactic automorphisms. ${ }^{2}$

We turn now to the central notion of a universal invariant. To say that the property of being an anaphor is a universal (invariant) says that in each adequate grammar $\mathrm{G}$ for a natural language the (possibly empty) set of anaphors in $\mathrm{L}(\mathrm{G})$ is fixed by all the (stable) automorphisms of G. Crucial here: different G's may build expressions in quite different ways - we just claim that the maps that respect how they build expressions always map anaphors to anaphors. Of course for this claim to make sense we need (and have) a language independent definition of anaphor.

\section{Anaphora}

We offer, unsurprisingly, a semantically based definition. An expression whose semantic value $\mathrm{F}$ maps binary relations to unary ones (or more generally $n+2$-ary ones to $n+1$-ary ones) often satisfies the AEC (Accusative Extensions Condition) below:

(AEC) If the set of things that an object $a$ stands in the relation $\mathrm{R}$ to is the same set that $b$ stands in the relation $\mathrm{S}$ to, then $a \in \mathrm{F}(\mathrm{R})$ iff $b \in \mathrm{F}(\mathrm{S})$.

For example most poets satisfies the AEC: if Sue admires just the objects that John distrusts then Sue admires most poets and John distrusts most poets have the same truth value (both true, or both false). But simple reflexives in English, like himself or herself, as well as more complex ones, such as every student but himself, only herself and the teacher clearly fail the AEC. If John criticized just Sue, Bill, and Trevor and these are exactly the objects that Bill praised, then John criticized himself is false, but Bill praised himself is true. But these reflexive "anaphors" do satisfy the weaker AAC (Accusative Anaphor Condition) below:

(AAC) If the set of things that $a$ stands in the relation $\mathrm{R}$ to is the same set that $a$ stands in the relation $\mathrm{S}$ to then $a \in \mathrm{F}(\mathrm{R})$ iff $a \in \mathrm{F}(\mathrm{S})$.

\footnotetext{
${ }^{2}$ Keenan and Stabler (2003, 65-66) generalize invariants to those linguistic objects preserved by stable automorphisms, but for present purposes the simpler notion defined here suffices.
} 
So if John praised just the objects he admires then he praised himself (or everyone but himself) iff he admires himself (or everyone but himself). And we now define:

(1) An anaphor is an expression that denotes a mapping that satisfies the AAC but in some models fails the AEC.

We can now test non-circularly whether anaphors in a given language have one or another syntactic property. And note that our definition does not imply that all languages have anaphors, nor does it require that anaphors be NPs/DPs rather than clitics or verbal affixes.

\subsection{Voice conditioned anaphora: Toba Batak}

Toba Batak in (2) illustrates conditioning of the distribution of anaphors by verb voice, a common pattern in W. Austronesian (Tagalog, Balinese, Malagasy). The constituent structure we give draws on Schachter (1984), Cole and Hermon (2008) and Keenan (2009), and is summarized in Keenan and Stabler (2003, 65-66). Morphemes mang- and di- are prefixes, forming transitive verbs from roots.

$$
\begin{aligned}
& \text { a. [Mang+ida dirina }] \text { [si Torus }] \\
& \text { AF }+ \text { see self ART Torus }
\end{aligned}
$$

'Torus saw himself'

$$
\begin{array}{llll}
\text { b. } & \text { [Di+ida } & \text { [si Torus }] & \text { dirina }] \\
\text { PF }+ \text { see } & \text { ART Torus } & \text { self }
\end{array}
$$

'Torus saw himself'

In (2b) the anaphor dirina asymmetrically c-commands its proper name antecedent si Torus, contra Principle (A). But the crucial property of (2) is not inconsistency with (A), it is that the distribution of anaphor and antecedent is characterizable in terms of verbal morphology: mangverbs may combine with an anaphor forming a unary predicate whose argument may antecede the anaphor but can not itself be one. $d i$ - prefixed transitive verbs may combine with non-reflexive DPs $x$ to form unary predicates taking an anaphor as argument anteceded by $x$. Keenan and Stabler $(2003,71-72)$ present a compositional interpretation for $(2 a, b)$ which uses the verbal morphology in a natural way. So Toba speakers learn morphology that has little analogue in English. That is, Toba and English are structurally different.

In fact, it is easy to see that the voice markers mang- and $d i$ - play a special role in the grammar, and so they are structural in the sense defined above. That is, we cannot trade either one of these elements in for any other expressions without disrupting at least some of the derivations in the language. This is argued in detail in Keenan and Stabler $(2003, \S 2.3)$ and established conclusively for a small illustrative Toba Batak grammar.

\subsection{Case conditioned anaphora: Korean}

(3) illustrates a common paradigm in which the two arguments of a transitive verb are distinctively case marked. (Commonly one of the two would be replaced by the "topic" marker $-(n) u n$ in main clauses).

(3) a. John-i Sam-ul piphanhayssta 'John criticized Sam'

b. Sam-ul John-i piphanhayssta 'John criticized Sam' 
The preverbal linear order of arguments is quite free, their case markers $-i$ and $-u l$ identify their argument roles. Interchanging them results in interchanging the argument roles as well, the result translating as 'Sam criticized John'. Preverbal word order freedom is maintained with reflexives:
a. John-i
John
caki-casin-ul piphanhayssta
self-EMPH
criticized
b. caki-casin-ul John-ul
piphanhayssta
self-EMPH
John
criticized

(4b) has no natural constituent structure analysis in which the -ul marked argument is asymmetrically c-commanded by its antecedent. On our analysis it asymmetrically c-commands its antecedent. (Keenan and Stabler $(2003, \S 2.1)$ show how to make the two arguments and the transitive verb sisters). This word order freedom remains when the antecedent is quantified, as in (motun) haksayng-tul-i '(all) the students', or nwuka 'Who?'. In these cases and (4a,b), speakers reject interchanging the case markers as in (5), a fact also noted in Park (1986, p.244).

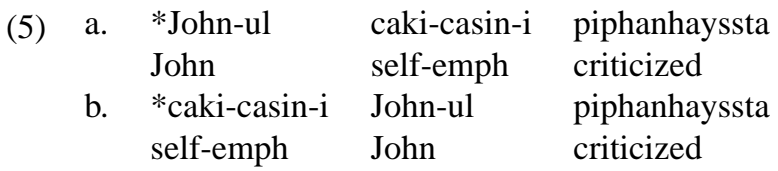

Thus to interpret transitive sentences like those in (4) correctly and to avoid overgeneration (5a,b), we must mention specific case markers (such as $-i /-k a$ and $-(l) u l$ ). This says that case markers are an integral part of syntactic structure and are semantically interpreted. Learners of Korean must attend to a property of language which is only marginal in English and not present at all in Toba Batak, namely, overt nominal case marking.

In Korean, it is easy to see that the case markers play a special role in the grammar, and so they are structural in the sense defined above. That is, we cannot trade a case marker in for any other expressions without disrupting at least some of the derivations in the language. This is argued in detail in Keenan and Stabler $(2003, \S 2.1)$ and established conclusively for a small illustrative Korean grammar.

\subsection{Voice and case conditioned anaphora: Tagalog}

Tagalog conditions the distribution of anaphors using both verbal and nominal morphology. Contrast (6a), formed by infixing the root with -in- and (6b), which infixes -um-:
a. Sinampal ng
guro ang
estudyante
praised
teacher
student
'The teacher slapped the student'
b. Sumampal ng estudyante ang guro
praised student
teacher

'The teacher slapped the student'

The postverbal order of the full DPs ng guro and ang estudyante in (6a) is fairly free (Schachter and Otanes, 1972, p.83), (Kroeger, 1993, p.110), but very unlike postverbal order in Toba Batak. But if we merely interchange $n g$ and ang in (6a) without changing the verbal infixes -in- and -um-, we change the meaning to 'The student slapped the teacher' (similarly for (6b)). As with the external DPs in Toba Batak, only ang DPs can be relativized. Constraints on the distribution of reflexives are comparable to those in Korean and Toba, the crucial cases being $(7 a, b)$. 
(7)

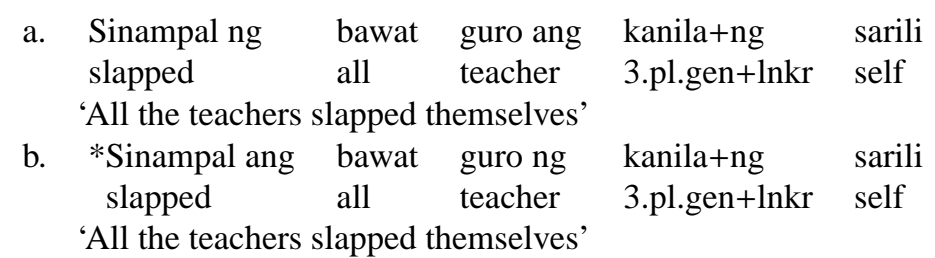

Interchanging the prenominal case markers in (7a) results in the strongly ungrammatical (7b). So verbal morphology conditions the interpretation of case marked arguments and the distribution of anaphors and so is an integral part of syntactic and semantic structure.

\subsection{Two universals of binding theory.}

If we provide grammars that straightforwardly generate the analyses sketched above, with natural semantics, the anaphors are not generally c-commanded by their antecedents, strongly suggesting that (A), as interpreted here, is false. But reflecting on these grammars, the anaphors in all of these languages, and in English too, have some striking common properties! In particular, in all of these langauges, the anaphors play a special role in the grammar: they have special properties with respect to case marking or voice marking or linear position which make them different from all other expressions. So we claim

U1. In all human languages, the set of anaphors is invariant.

In English, the special structural properties are manifest in the way they combine with transitive verbs. In Toba Batak, the anaphors interact with the voice system in a distinctive way, and in Tagalog, the anaphors interact with the voice and case system in a distinctive way. Keenan and Stabler (2003) use simple illustrative grammars to show how the grammars determine what items are invariant, how the anaphors are determined by the semantics, and how it then becomes a demonstrable fact that, for the languages as defined by these grammars, the anaphors are invariant. Our empirical claim is that this will hold in all languages.

The relations between anaphors and antecedents are also similar across all these languages, in spite of the fact that the languages are structurally quite diverse. In particular, the relation between anaphors and the elements that can antecede them is a structural one in all these languages, and so we propose

U2. In all human languages, the is a possible antecedent of relation is invariant.

Again, once the grammars are given, the question of whether this is true or not is determined.

\section{Conclusions}

We have proposed two binding theory universals, formulated in such a way that they can apply to languages which are not structurally the same "at any level of structure." So these new universals, like the ones mentioned in $\S 2$, are substantial, falsifiable, and compatible with very considerable diversity. It is reasonable to expect that more universals of this kind will be discovered, providing a universal grammar that is rich and substantial but nevertheless compatible with the enormous diversity of human langauges. 


\section{References}

I. Asimov, Understanding Physics, Volume 1, New American Library, NY, 1966.

D. Büring, Bound to bind, Linguistic Inquiry 36 (2) (2005) 259-274.

N. Chomsky, Aspects of the Theory of Syntax, MIT Press, Cambridge, Massachusetts, 1965.

P. Cole, G. Hermon, VP raising in a VOS language, Syntax 11 (2) (2008) 144-197.

S. Crespi-Reghizzi, G. Guida, D. Mandioli, Noncounting context free languages, Journal of the Association for Computing Machinery 25 (4) (1978) 571-580.

N. Evans, S. Levinson, The myth of language universals: Language diversity and its importance for cognitive science, Behavioral and Brain Sciences 32 (2009) 429-448.

G. Frege, Gedankengefüge, Beträge zur Philosophie des deutschen Idealismus 3 (1923) 36-51, translated and reprinted as 'Compound thoughts' in Mind 72(285): 1-17, 1963.

A. Joshi, How much context-sensitivity is necessary for characterizing structural descriptions, in: D. Dowty, L. Karttunen, A. Zwicky (Eds.), Natural Language Processing: Theoretical, Computational and Psychological Perspectives, Cambridge University Press, NY, 206-250, 1985.

Y. Kaji, R. Nakanishi, H. Seki, T. Kasami, The Computational Complexity of the Universal Recognition Problem for Parallel Multiple Context-Free Grammars, Computational Intelligence 10 (1994) 440-452.

M. Kanazawa, A pumping lemma for well-nested multiple context free grammars, Tokyo University, forthcoming, 2009.

E. L. Keenan, Voice determines co-argument anaphora in W. Austronesian, in: S. Chung, D. Finer, I. Paul, E. Potsdam (Eds.), Proceedings of the XVIth Annual Meetings of the Austronesian Formal Linguistics Association (AFLA XVI), 77-91, http://ling.uwo.ca/meetings/afla16/proceedings.htm, 2009.

E. L. Keenan, E. P. Stabler, Bare Grammar: Lectures on Linguistic Invariants, CSLI Publications, Stanford, California, 2003.

E. L. Keenan, E. P. Stabler, Linguistic invariants and language variation, in: P. Hajek, L. Valdes-Villanueva, D. Westerståhl (Eds.), Logic, Methodology and Philosophy of Science, King's College Publications, London, 395-411, 2005.

G. M. Kobele, Generating Copies: An Investigation into Structural Identity in Language and Grammar, Ph.D. thesis, UCLA, 2006.

E. König, V. Gast (Eds.), Reciprocals and reflexives : theoretical and typological explorations, Mouton de Gruyter, NY, 2008.

P. Kroeger, Phrase Structure and Grammatical Relations in Tagalog, CSLI, Stanford, California, 1993.

J. Michaelis, M. Kracht, Semilinearity as a syntactic invariant, in: C. Retoré (Ed.), Logical Aspects of Computational Linguistics, Springer-Verlag (Lecture Notes in Computer Science 1328), NY, 37-40, 1997.

F. J. Newmeyer, Possible and Probable Languages: A Generative Perspective on Linguistic Typology, Oxford University Press, Oxford, 2005.

S.-H. Park, Parametrizing the Theory of Binding: The Implications of caki in Korean, Language Research 22 (1986) 229-253.

O. Rambow, Formal and Computational Aspects of Natural Language Syntax, Ph.D. thesis, University of Pennsylvania, computer and Information Science Technical report MS-CIS-94-52 (LINC LAB 278), 1994.

H. Seki, T. Matsumura, M. Fujii, T. Kasami, On multiple context-free grammars, Theoretical Computer Science 88 (1991) 191-229.

E. P. Stabler, Derivational minimalism, in: C. Retoré (Ed.), Logical Aspects of Computational Linguistics, SpringerVerlag (Lecture Notes in Computer Science 1328), NY, 68-95, 1997.

P. Schachter, Studies in the Structure of Toba Batak, Tech. Rep. UCLA Occasional Papers in Linguistics, Number 5, UCLA, Los Angeles, 1984.

P. Schachter, F. T. Otanes, Tagalog Reference Grammar, University of California Press, Los Angeles, 1972. 